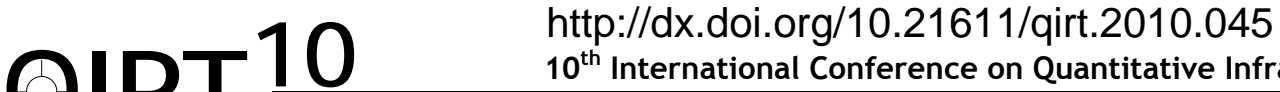 \\ $10^{\text {th }}$ International Conference on Quantitative InfraRed Thermography \\ July 27-30, 2010, Québec (Canada)
}

\section{A novel Infrared Thermography (IRT) based experimental technique for distributed temperature measurements in hot gas flows}

by M. Gallo*, J. P. Kunsch and T. Rösgen

Institute of Fluid Dynamics, ETH Zürich, Zürich 8092, Switzerland.

*Corresponding author. E-mail: gallo@ifd.mavt.ethz.ch, tel.:+41 4463209 18, fax: +41 446321147.

\begin{abstract}
A new experimental technique is being developed to overcome the drawbacks exhibited by existing techniques used to perform temperature measurements in flow fields. It requires the insertion into the flow of a high porosity grid formed by a thin electrically conductive wire. By measuring the grid temperature distributions with an infrared (IR) camera, one can calculate both, the distribution of the convective heat transfer coefficient and the local fluid temperature. In this paper the physical principles of the technique, the calibration of the IR camera and the experimental procedure will be described. As a initial test case the technique is used to measure the fluid temperature profiles in a heated free jet.
\end{abstract}

\section{Introduction}

The knowledge of the temperature distribution in a flow field can be of fundamental importance to understand the underlying physical processes. Currently, there are several techniques available which can be used for such investigations. Unfortunately all of these techniques are affected by different drawbacks.

The temperature distributions in a flow field can for example be recorded by thermocouples. While they are capable of providing quite accurate measurements, the surveys can be quite time consuming and intrusive. Non-intrusive temperature measurements can be carried out by laser-based techniques (LIF/PLIF) which are affected by high equipment cost and general complexity of operation. The use of infrared thermography to perform direct temperature measurements in a gas flow provides reliable results only in cases of high emissivity such as in yellow flames or for hot combustion products containing substances $\left(\mathrm{CO}_{2}\right.$ and $\left.\mathrm{H}_{2} \mathrm{O}\right)$ which emit in a suitable infrared band. The technique to visualize and to measure temperature distributions of a gas flow using IR thermography and a high emissivity mesh inserted into the flow, was initially proposed by Anderson et al. [1] and Burch et al. [2]. More recently Cehlin et al. [3] have suggested a variant of the technique. They placed into the flow a paper screen to investigate the temperature fields by means of IR thermography. Although it provides a global view of the temperature field, the technique cannot be employed for the exploration of a hot gas flow field. Neely [4] defined the physical and geometric characteristics of a mesh so as to render it resistant to high temperatures. In addition, he investigated under which conditions the mesh surface temperature depends only on the local gas temperature. Despite its small upstream influence, a transverse mesh is responsible for a strong downstream impact resulting both in the formation of wake structures and in the alteration of the turbulence levels. In order to make the sensors (meshes) described in the previous studies less intrusive, a new technique has been developed using a planer array formed by a "meandering" wire. This wire, which represents several loops, allows the mapping of the temperature of a hot gas flow with an acceptable spatial resolution. Following the basic idea originally proposed by Gartenberg and Roberts [5], the technique described in the present paper may also be used to measure the distribution of the velocity component normal to the plane of the grid.

An objective of the present work is to describe the basic physical principles which govern the new experimental technique. Subsequently the calibration procedure of the IR camera and the experimental procedure will be described. In order to validate the new experimental technique, temperature profiles were acquired in a free hot jet using the new technique and a conventional rake of thermocouples at the same locations.

\section{Heated grid technique}

This technique allows to perform measurements in a plane of both the temperature and the main velocity, i. e. the velocity normal to the plane. It requires the insertion into the flow field of a very high porosity grid (the sensor), normal to the main flow direction, formed by a thin electrically conductive wire having high thermal emissivity. The sensor surface temperatures are measured by means of an infrared (IR) camera. 


\subsection{Governing equations}

Eq. 1 represents the energy balance for an annular element of a long solid cylinder $(L>>r)$ with internal heat generation (Fig. 1). In this case the heat is generated as the result of the current passage in the wire of the sensor (Joule effect).

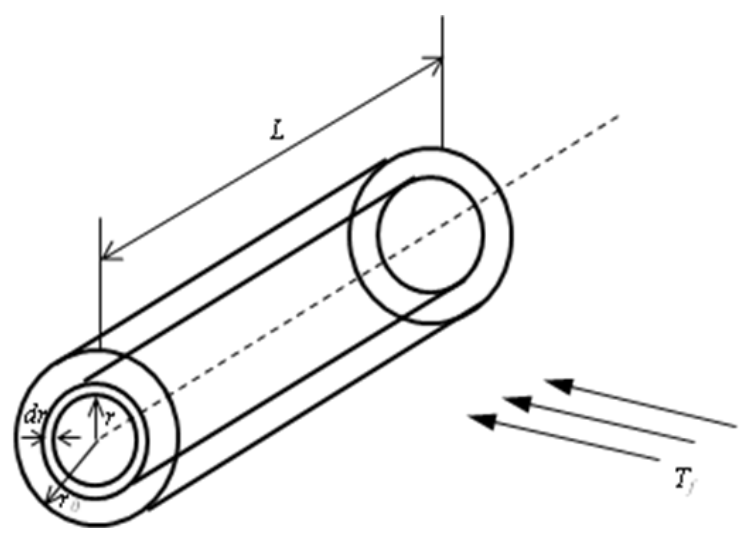

Fig. 1. Circular cylinder with internal heat generation

$$
q_{G} r=-k \frac{d}{d r}\left(r \frac{d T}{d r}\right)
$$

When the solid cylinder is immersed in a fluid having a temperature $T_{f}$, the boundary conditions require that the heat flux by conduction from the cylinder equals the rate of the convection and radiation flux at the surface:

$$
\begin{gathered}
-\left.k \frac{d T}{d r}\right|_{r=r_{0}}=\bar{h}_{c}\left(T_{0}-T_{f}\right) ; \\
T\left(r=r_{0}\right)=T_{0}
\end{gathered}
$$

Here, $h_{c}$ and $T_{0}$ are the heat transfer coefficient and the temperature at the specified surface $r=r_{0}$, respectively. By using the boundary conditions above, it is possible to obtain the temperature distribution in the cross section of the wire:

$$
T(r)-T_{f}=\frac{q_{G} r_{0}}{4 \overline{h_{c}}}\left\{2+\frac{\overline{h_{c}} r_{0}}{k}\left[1-\left(\frac{r}{r_{0}}\right)^{2}\right]\right\}
$$

The ratio $\frac{\overline{h_{c}} r_{0}}{k}$ represents the Biot number $(B i)$ which captures the relative importance of the conductive thermal resistance $R=r_{0} / k$ and the convective resistance $R_{C}=1 / \bar{h}_{C}$. If the Biot number approaches zero, the solid can be considered isothermal in the section normal to the longitudinal axis of the wire and the temperature change occurs primarily at the fluid-solid interface. When the Biot number is significantly smaller than one, eq. 2 can be rewritten ${ }^{1}$.

\footnotetext{
${ }^{1}$ Eq. 3 can be equally obtained by performing an overall energy balance: $q_{G}\left(\pi r_{0}^{2} L\right)=\bar{h}_{c}\left(2 \pi r_{0} L\right)\left(T-T_{f}\right)$
} 


$$
\frac{q_{G} r_{0}}{2}=\bar{h}_{c}\left(T-T_{f}\right)
$$

The heat transfer coefficient $\overline{h_{C}}$ includes both, the convective $\left(h_{c}\right)$ and radiative $\left(h_{r}\right)$ heat transfer contributions,

$$
\bar{h}_{c}=\left(h_{c}+h_{r}\right)
$$

Substituting eq. 4 into eq. 3 and imposing $T=T_{w h}$ leads to

$$
\frac{q_{G} r_{0}}{2}=h_{c}\left(T_{w h}-T_{f}\right)+h_{r}\left(T_{w h}-T_{f}\right)
$$

with

$$
h_{r}=\frac{\sigma \varepsilon\left(T_{w h}^{4}-T_{a m b}^{4}\right)}{\left(T_{w h}-T_{f}\right)}
$$

The temperature $T_{a m b}$ is the ambient temperature in case of unconfined flows, while for the confined ones $T_{a m b}$ is the bulk temperature $T_{b}$. Taking into account the expression of $h_{r}$ (eq. 6), eq. 5 becomes

$$
\frac{q_{G} r_{0}}{2}=h_{c}\left(T_{w h}-T_{f}\right)+\sigma \varepsilon\left(T_{w h}^{4}-T_{a m b}^{4}\right)
$$

In absence of heat generation $\left(q_{G}=0\right)$ eq. 7 can be written by setting $T=T_{w c}$

$$
O=h_{c}\left(T_{w c}-T_{f}\right)+\sigma \varepsilon\left(T_{w c}^{4}-T_{a m b}^{4}\right) .
$$

Subtracting eq. 7 from 8 the convective heat transfer coefficient $h_{c}$ can be evaluated using the following equation:

$$
h_{c}=\frac{1}{\left(T_{w h}-T_{w c}\right)}\left[\frac{q_{G} r_{0}}{2}-\sigma \varepsilon\left(T_{w h}^{4}-T_{w c}^{4}\right)\right]
$$

The sensor temperature distributions obtained by the IR camera relative to the heated case $\left(q_{G}>0, T=T_{\text {wh }}\right)$ and to the un-heated case $\left(q_{G}=0, T=T_{w c}\right)$ allow to evaluate the convective heat transfer coefficient distribution $\left(h_{c}\right)$ by means of eq. 9 . Once $h_{c}$ is known, eq. 7 or 8 yields the fluid temperature distributions $T_{f}$. This information can then be used to obtain a distribution of the local Nusselt number that is further linked, through King's law, to the local distribution of the Reynolds number and ultimately the mean flow velocity.

\subsection{Experimental set-up}

The experimental layout is depicted in Fig. 2. A single Cu-Ni wire with a diameter of $0.4 \mathrm{~mm}$ and coated with a very thin layer of high emissivity paint is employed to measure the fluid temperature profiles of a free hot jet. The wire is connected to a stabilized DC power supply, and the power input to the wire is monitored by precisely measuring voltage drop and current. The hot jet is generated by a heater-blower which creates a jet having a core with temperatures up to $700^{\circ} \mathrm{C}$. The temperature of the heated jet is kept constant during the tests by means of a PID controller that uses the signal of a thermocouple to control the power supplied. A second PID unit controls the blower which supplies the electric heater with air. It receives the input signal from a volumetric flow rate sensor and keeps the volume flow rate of the cold air supply constant. 
A circular pipe with a diameter $(D)$ of $6 \mathrm{~cm}$ is mounted immediately behind the heater in order to reach a full homogenization of the flow conditions at the exit section. The wire temperatures required to evaluate the fluid temperature are measured using an infrared camera with a mid-wave infrared (MWIR) InSb sensor $(3-5 \mu \mathrm{m})$ and 320x240 pixel resolution. A 50mm lens is used during the test at a distance of about $1.3 \mathrm{~m}$. To validate the present technique the temperature profiles are compared with those acquired at the same positions and flow conditions using a conventional rake of thermocouples of type $\mathrm{K}$.

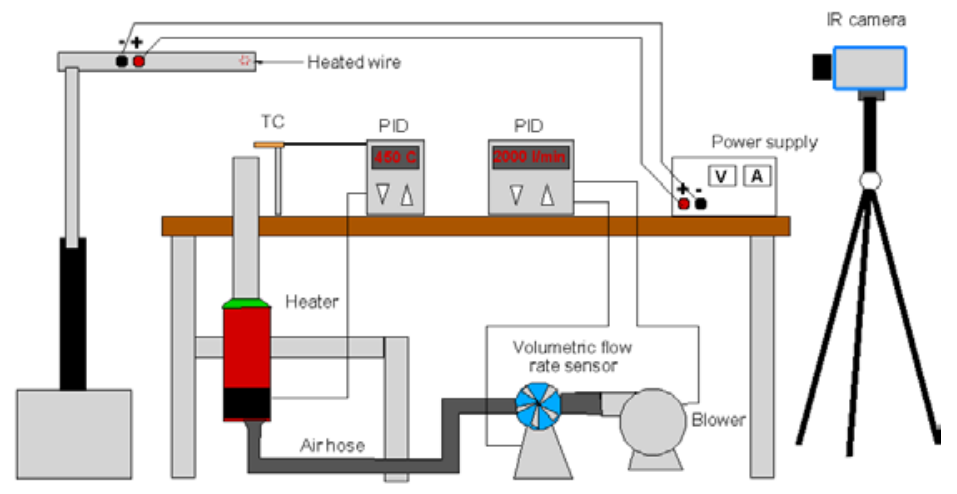

Fig. 2. Experimental setup

\subsection{Calibration procedure}

The goal of the calibration procedure is to determine a parametric mapping function which relates the wire surface temperature to the corresponding Digital Levels $(D L)$ of the IR camera. Owing to the small wire diameter and to the distance between the grid and the camera the single wire only partially covers the pixel surface (Fig. 3). The diagram presented in Fig. 4 shows the profile of the $D L$ of the wire heated by the power supply in quiescent air. The dashed line represents the wire $D L$ distributions obtained by recording the maximum digital levels $\left(D L_{\max }\right)$ seen in the pixels "crossed" by the wire. Although the wire temperature is constant, the curve exhibits an oscillating signature with a constant period. This behaviour is due to a slight misalignment of the wire with respect to the pixel rows of the sensor. The portion of a pixel covered by the wire changes with a period dependent on the angle formed between the wire and the sensor pixel rows. A better (near constant) trend is obtained by summing the $D L$ in the pixel crossed by the wire and the two $D L s$ of the vertical neighbours (see solid line Fig. 4). So a unique value of the $D L$ can be associated to each value of the wire temperature.

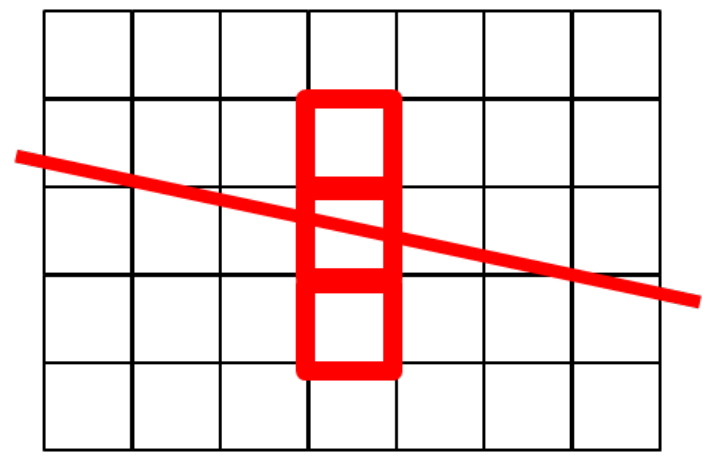

Fig. 3. Wire position relative to the pixels of the IR sensor

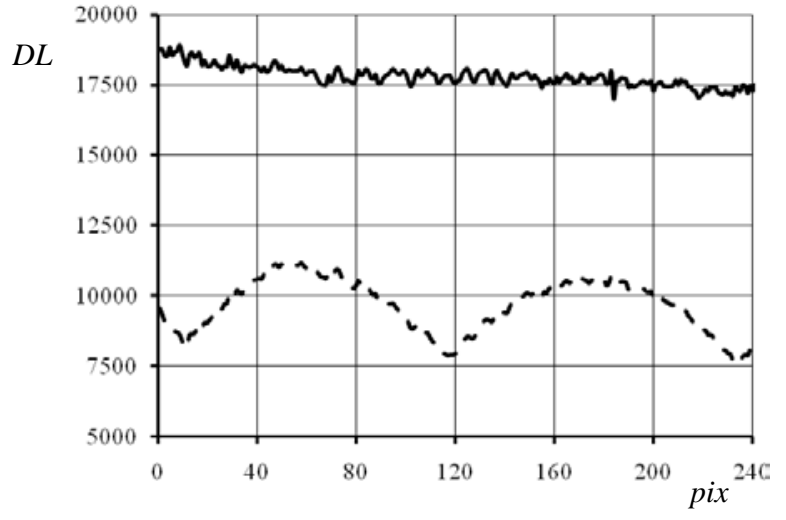

Fig. 4. DL profiles of the heated wire in quiescent air: profile of the maximum DLs (dashed line); profile of the sum of the DLs provided by three neighboring pixels vertically oriented (solid line)

For the calibration procedure the wire has to be positioned at about $1 \mathrm{~cm}$ from the nozzle exit, close to the thermocouple that while providing the signal for the PID controller measures the fluid temperature $\left(T_{f_{-} T C}\right)$ immediately behind the nozzle exit. The mass flow rate is kept constant by the PID controller which drives the blower used for the air supply to 
the heater. During the calibration procedure it is necessary to vary the fluid temperature while the mass flow rate must be constant. For each value of the temperature, the $D L$ of the unheated $(Q=0)$ and heated wire $(Q>0)$ have to be acquired (Fig.5). The calibration procedure relies on a parametric model (quadratic polynomial with coefficients $p_{k}$ ) for the relationship between $D L$ and wire temperature $T_{w}$,

$$
T_{w}=f\left(D L,\left\{p_{k}\right\}\right)
$$

An iteration scheme is implemented which minimizes the error (eq. 11) between the true flow temperature $\bar{T}_{f \_T C}$ as measured by the thermocouple and the estimated value $\bar{T}_{f \_l R}$, as obtained with the temperature model described in section 2.1. Indeed the wire temperatures for the cold $T_{w c}$ and heated case $T_{w h}$, evaluated by eq. 10, are used to calculate the convective heat transfer coefficient $h_{c}$ (eq. 9) and by using eq. 7 or 8 the fluid temperature $\bar{T}_{f \_I R}$.

$$
\min _{\left\{p_{k}\right\}} \varepsilon=\sum_{i=1}^{n} \sqrt{\left(\bar{T}_{t-T C}^{(i)}-\bar{T}_{t-I R}^{(i)}\right)^{2}}
$$

The individual calibration wire temperatures $\bar{T}_{w}^{(i)}$ are obtained by averaging the $D L s$ over the uniformly heated section of the wire. An example of the resulting $D L$ calibration curve is shown in Fig. 6.

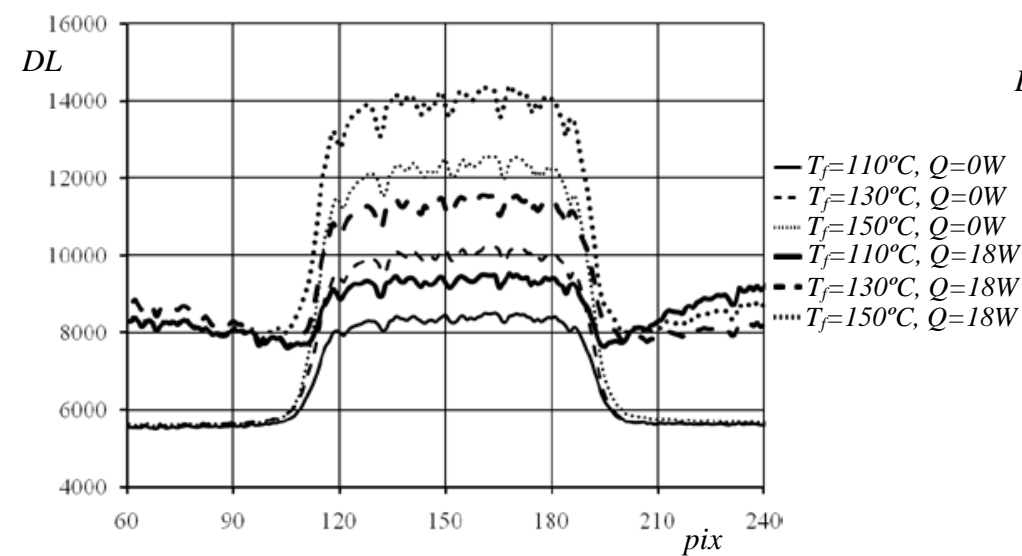

Fig. 5. Examples of $D L$ profiles acquired during the calibration procedure

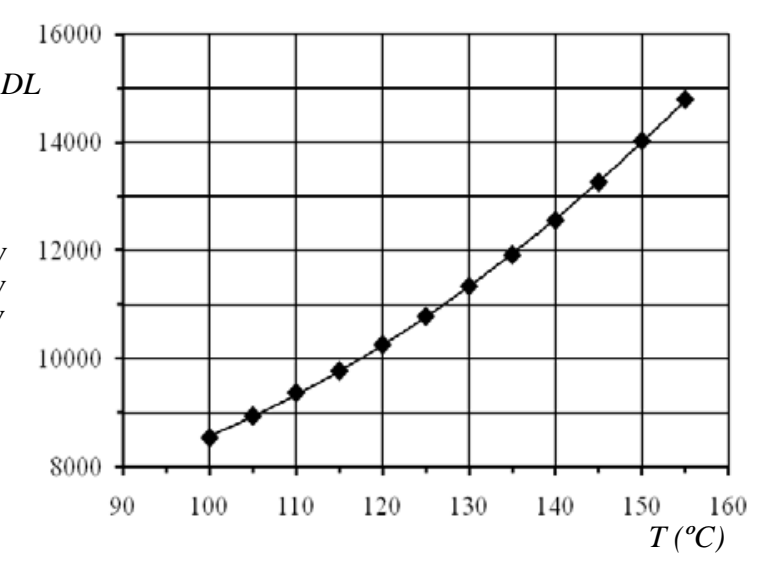

Fig. 6. Example of calibration curve

\subsection{Experimental procedure for the fluid temperature measurements}

To measure the fluid temperature profile it is necessary to acquire the $D L$ distributions for two different heating levels of the wire immersed into the flow field. In the following figures both the thermal images (Fig. 7) and the corresponding $D L$ profiles (Fig. 8) are reported.

The $D L$ profiles shown in Fig. 8 can be converted into temperatures by using the calibration curve recorded with the same integration time as the one used for the image acquisition during the actual tests. In this case the integration time was $60 \mu \mathrm{s}$, with an associated temperature range from 185 to $250^{\circ} \mathrm{C}$. The wire temperature profiles corresponding to the two heating levels are used to evaluate the convective heat transfer coefficient distributions $h_{c}$ (eq. 9) and by eq. 7 or 8 the fluid temperature profile is obtained (Fig. 9). 


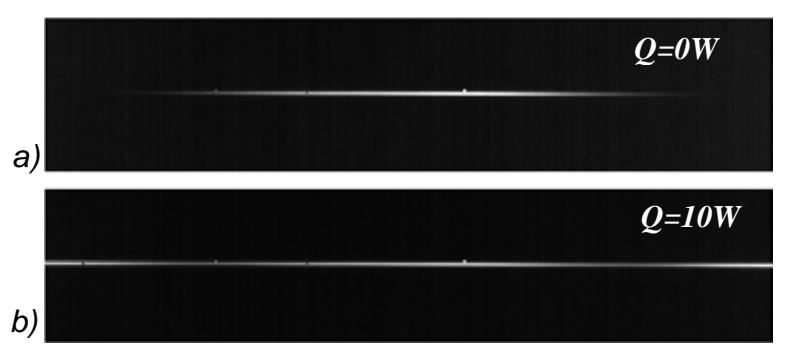

Fig. 7.Thermal images of the wire: a) unheated case; b) heated case

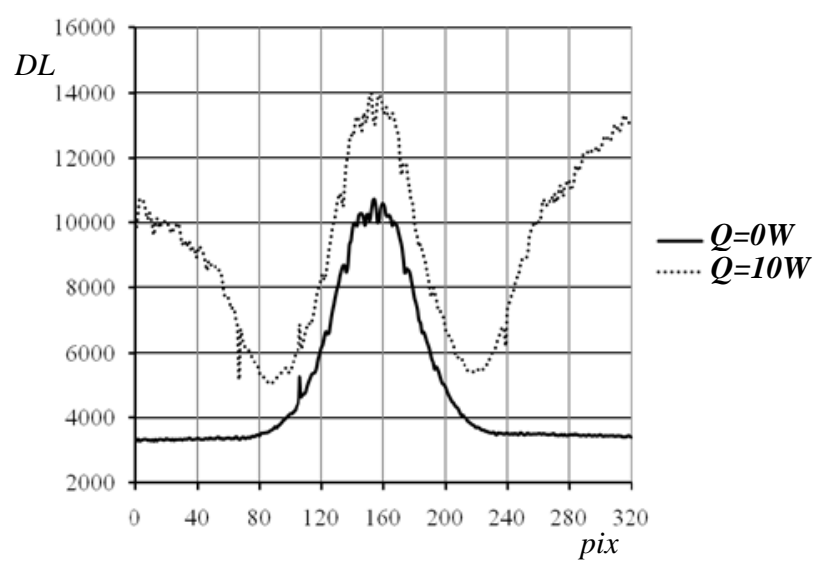

Fig. 8. DL profiles relative to the unheated (solid line) and heated case (dotted line)

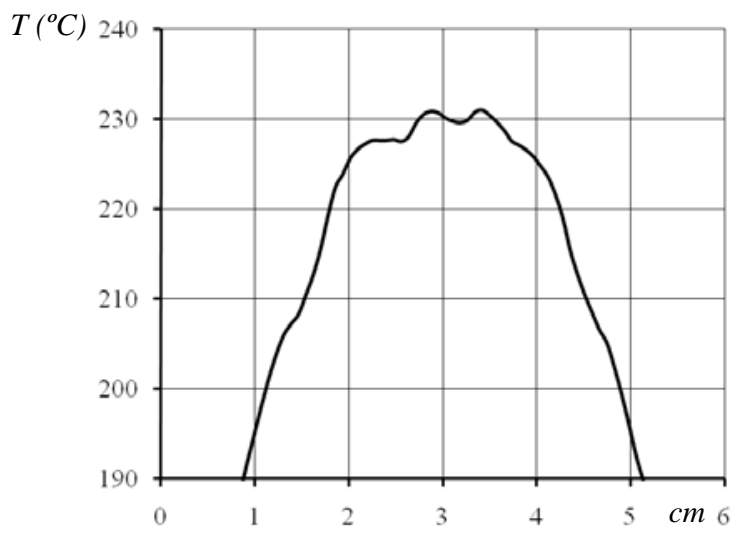

Fig. 9. Temperature profile

\section{Fluid temperature measurements}

The feasibility of the heated grid technique is demonstrated by measuring the temperature field of a free hot jet. The axis of the jet is vertical to ensure full symmetry w.r.t. its axis. The Reynolds number, based on the diameter D of the exit section, is 32000 and the gas temperature is $230^{\circ} \mathrm{C}$. The wire used for the heated grid technique is placed at four different heights from the exit of the nozzle, i.e. at $x / D=3.33,5.00,6.67$ and 8.33. A rake of thermocouples is placed at the same locations $x / D$ from the nozzle exit in order to obtain temperature data for reference. A single integration time is not sufficient to establish a calibration curve valid for the whole temperature range of the jet. Hence, the thermal images of the wire have been acquired for different integration times depending on the maximum temperature of the jet and thus on the distance from the nozzle. By means of the different calibration curves, corresponding to different integration times, fluid temperature profiles, with temperatures ranging from $75^{\circ} \mathrm{C}$ to $250^{\circ} \mathrm{C}$, could be reconstructed. The results are summarized in Fig. 10 showing the temperature profiles obtained using the heated grid technique (solid lines) and thermocouples (points). The latter are shown for validation purposes. In addition, a curve fit using a Gaussian profile was applied to the thermocouple measurements to extract the basic data characterizing the aero-thermodynamic features of the jet. It is clearly visible from Fig.10 that the maximum temperature measured at the centerline of the jet decreases with increasing distance from the nozzle exit, while the width increases. It can be observed for each $x / D$ that the profiles acquired with the rake of thermocouples and the heated grid technique are in good agreement in the region close to the jet axis, where the temperatures exceed $120^{\circ} \mathrm{C}$. In proximity to the jet boundaries the discrepancy between both measuring techniques becomes more apparent, increasing with the distance from the nozzle exit. The long integration times required for low temperature measurements and the corresponding reduction in the signal-to-noise ratio may be one reason for this discrepancy. 


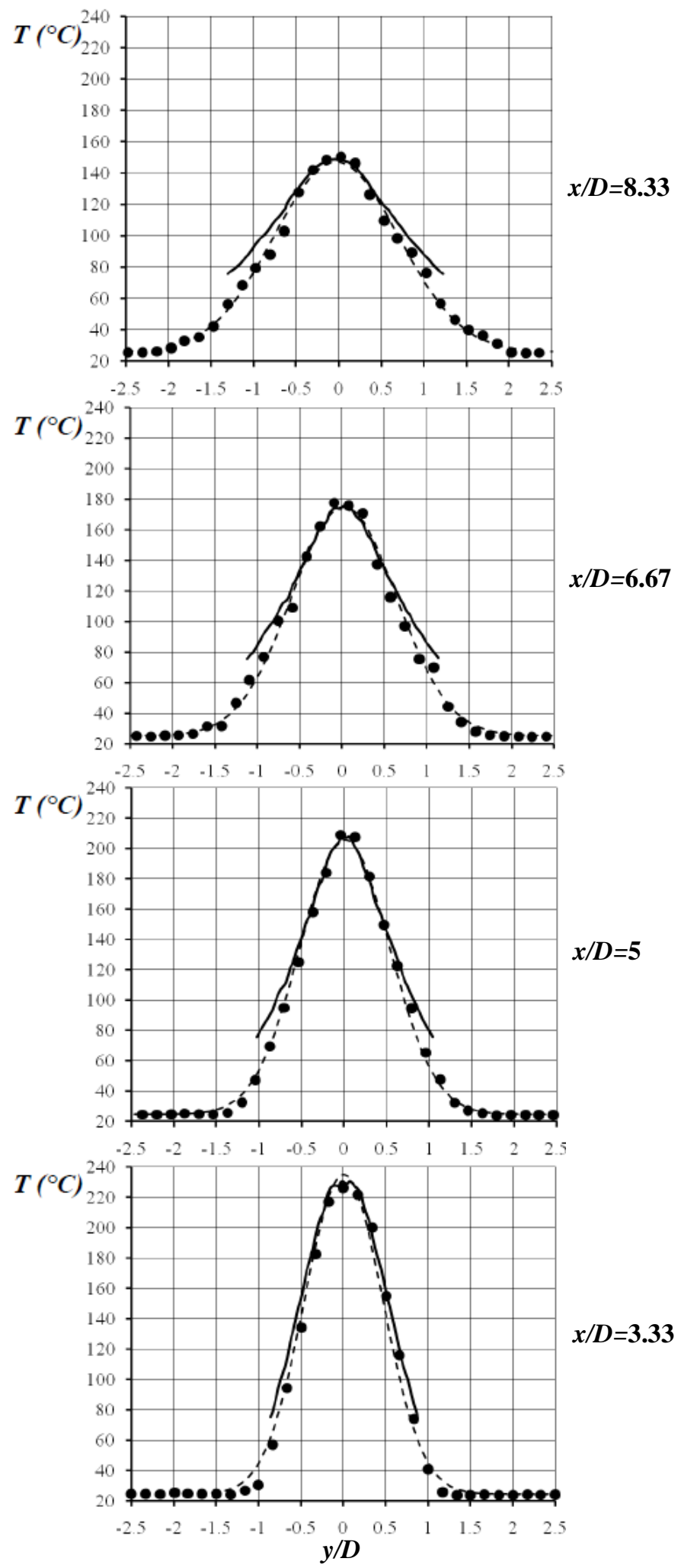

Fig. 10. Temperature profiles acquired for $x / D=3.33,5,6.67$ and 8.33: heated wire technique (solid line); thermocouple measurements (circular dots); Gaussian fit of the thermocouple measurements (dashed line.). 


\section{Conclusions}

In the present paper a new experimental technique for temperature measurements in a hot gas flow was introduced. The physical principles were outlined, and the calibration and experimental procedures were described in detail. The temperature field of a free hot gas jet was investigated utilizing the new technique and conventional thermocouple measurements. The new technique could be validated successfully comparing the temperature profiles obtained by both techniques. In particular, for gas temperatures higher than $120^{\circ} \mathrm{C}$ the maximum error is less than $3 \%$.

\section{REFERENCES}

[1] Anderson R., Hassani V. and Kirkpartick. A., "Visualizing the air flow from cold air ceiling jets", ASHRAE Journal, vol. 33, p. 30-35, 1991.

[2] Burch S., Hassani V. and Penney T., "Use of infrared thermography for automotive climate control analysis", SAE paper no. 921136. Society of Automotive Engineers, Warrendale, 1992.

[3] Cehlin M., Moshfegh B. And Sandberg M., "Measurements of air temperatures close to a low-velocity diffuser in displacement ventilation using a infrared camera", Energy and Buildings, vol. 34, p. 687-698, 2002.

[4] Neely A. J., "Mapping temperature distributions in flows using radiating high-porosity meshes", Experiments in Fluids, vol. 45, p. 423-433, 2008.

[5] Gartenberg E., Roberts S. A., "Mapping flow fields with a heated wire and infrared imaging system", J. Aircraft, vol. 28, p. 284-286, 1991. 